\title{
Two families with autosomal dominant progressive external ophthalmoplegia
}

\author{
S Kiechl, R Horváth, P Luoma, U Kiechl-Kohlendorfer, B Wallacher-Scholz, R Stucka, C Thaler, \\ J Wanschitz, A Suomalainen, M Jaksch, J Willeit
}

J Neurol Neurosurg Psychiatry 2004;75:1125-1128. doi: 10.1136/jnnp.2003.025890

See end of article for authors' affiliations

Correspondence to: Dr S Kiechl, Department of Neurology, University of Innsbruck, Anichstraße 35, 6020 Innsbruck, Austria; stefan.kiech@ @ibk.ac.at

Received 13 August 2003 Revised

24 September 2003

Accepted 12 October 2003

\begin{abstract}
Objectives: We report here the clinical and genetic features of two new families with autosomal dominant progressive external ophthalmoplegia (adPEO).

Patients and methods: The examination of index patients included a detailed clinical characterisation, histological analysis of muscle biopsy specimens, and genetic testing of mitochondrial and nuclear DNA extracted from muscle and leucocytes.

Results: Index patients in both families presented with PEO and developed other clinical disease manifestations, such as myopathy and cardiomyopathy (patient 1) and axonal neuropathy, diabetes mellitus, hearing loss, and myopathy (patient 2), later in the course of illness. Both patients had ragged red fibres on muscle histology. Southern blot of mtDNA from muscle of patient 2 showed multiple deletions. In this case, a novel heterozygous missense mutation F485L was identified in the nuclear encoded putative mitochondrial helicase Twinkle. The mutation co-segregated with the clinical phenotype in the family and was not detected in 150 control chromosomes. In the other index patient, sequencing of ANT1, C10orf2 (encoding for Twinkle), and POLG1 did not reveal pathogenic mutations.

Conclusions: Our cases illustrate the clinical variability of adPEO, add a novel pathogenic mutation in Twinkle (F485L) to the growing list of genetic abnormalities in adPEO, and reinforce the relevance of other yet unidentified genes in m+DNA maintenance and pathogenesis of adPEO.
\end{abstract}

$\mathrm{P}$ atients with autosomal dominant progressive external ophthalmoplegia (adPEO) were first reported in the late 1980s. ${ }^{1}$ Apart from chronic progressive external ophthalmoplegia, adPEO patients may suffer from generalised myopathy and exercise intolerance. In addition, some adPEO patients develop clinical signs suggestive of multiorgan involvement such as ataxia, peripheral neuropathy, encephalopathy, depression, cataracts, hearing loss, cardiomyopathy, and endocrine dysfunction. ${ }^{2-5}$ Most patients show cytochrome c oxidase negative, ragged red fibres in muscle biopsy specimens, and multiple mtDNA deletions on Southern blot. ${ }^{6}$ On a molecular level, adPEO arises from defects in mtDNA maintenance, and underlying mutations have been identified in three nuclear encoded genes: $A N T 1,{ }^{8-10}$ C10orf2 (encoding for Twinkle), ${ }^{11-13}$ and POLG ${ }^{14-16}$ However some adPEO families do not show mutations in any of these genes, indicating further genetic heterogeneity. We report here the clinical features and genetic findings in two new families with adPEO.

\section{PATIENTS AND METHODS}

Clinical and electrophysiological findings, and muscle histology of the two index patients are summarised in table 1 . The pedigrees are shown in fig 1.

\section{Patient 1}

This 67 year old man had experienced slowly progressive external ophthalmoplegia from the age of 15 years. At 49 years of age, dyspnoea during exercise, fatigue, peripheral oedema, nocturia, and palpitations developed. Echocardiography showed an advanced dilated cardiomyopathy with marked reduction of ventricular wall movements and an ejection fraction of less than 25\%. Mild improvement was achieved with diuretics, digitalis, and ACE inhibitor treatment. Because of atrial fibrillation with periods of paroxysmal ventricular tachyarrythmia, anticoagulation was started and because of severe symptoms the patient was placed on the waiting list for heart transplantation. Neurological evaluation revealed ptosis, mild facial weakness, and limitation of eye movements in all directions. Electromyography of right biceps and facial muscles showed short duration, low amplitude voluntary motor unit action potentials and an early recruitment of many motor units indicative of a myopathy. The family history was remarkable for progressive bilateral ptosis and ophthalmoplegia in the patient's grandfather, mother (+ diabetes mellitus) and one of his two sons, but none had cardiac disease manifestation (fig 1 ).

\section{Patient 2}

This patient is a 68 year old woman. Ptosis and limited eye movements were noted at the age 50. Later on, she developed progressive atrophy and weakness of the shoulder and quadriceps muscles as well as exercise intolerance. More recently, she also showed axonal polyneuropathy, hearing loss, dysphagia, diabetes mellitus, and idiopathic osteoporosis. On neurological examination, she had almost complete bilateral ptosis and ophthalmoplegia. Her fundi were normal. There was a moderate weakness and atrophy of her quadriceps and shoulder muscles. She was able to walk, but had problems in climbing stairs and lifting her arms over the shoulder level. Achilles tendon reflexes were absent. Electromyography recording from shoulder muscles showed a prominent myopathic pattern. An MRI scan of the brain was unremarkable. The patient's mother had had PEO as an isolated clinical feature. One brother had suffered from PEO,

Abbreviations: adPEO, autosomal dominant progressive external ophthalmoplegia; CS, citrate synthase; NCP, non-collagen protein; RC, respiratory chain; RFLP, restriction fragment length polymorphism 


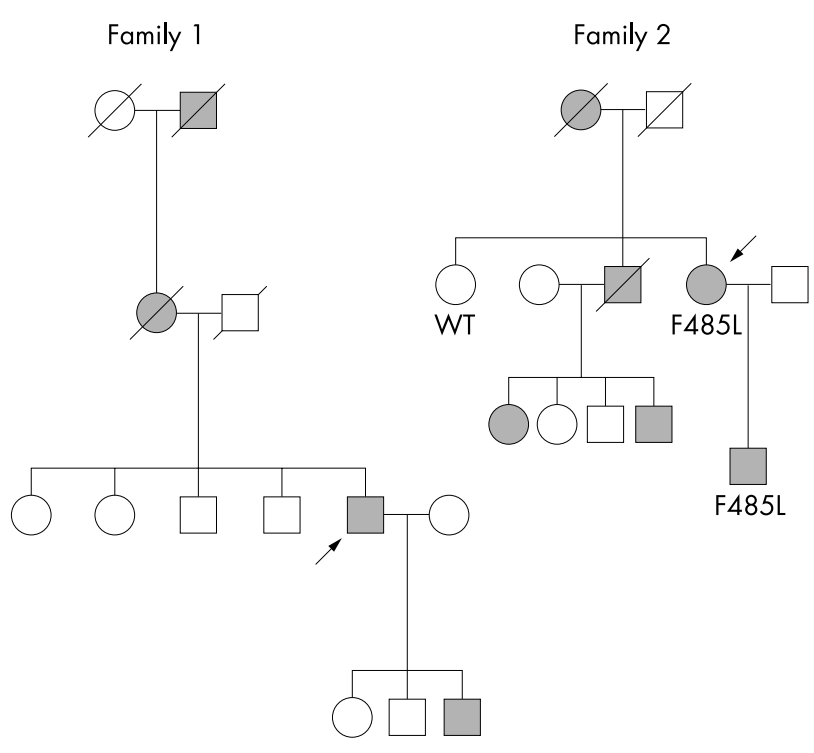

Figure 1 Family pedigrees. Solid symbols indicate clinically affected individuals. Barred symbols indicate deceased individuals. Arrows indicate the index patients. WT, wild type.

diabetes, cardiomyopathy, and myopathy of the respiratory muscles, and died from respiratory insufficiency. The patient's son and two children of her brother show incipient and isolated PEO.

\section{Morphology and biochemistry of skeletal muscle}

An open muscle biopsy was performed in both index patients (at age 55 and 65 years, respectively). Serial cross-sections (6 $\mu \mathrm{m}$ thick) were processed for histochemical stains according to standard procedures. A deep frozen part of the biopsy was used for biochemistry. Respiratory chain (RC) enzyme complex I-IV activities were determined in skeletal muscle as described. ${ }^{17}$ In patient 1 , muscle tissue was not sufficient for ultrastructural and biochemical analysis and a second biopsy was not considered because of long term anticoagulation.

\section{DNA analysis}

Total DNA was extracted from muscle and leucocyte samples using a commercial purification kit (Qiagen, Hildesheim, Germany). PCR, Southern blot and single strand conformational polymorphism analyses were performed as described previously. ${ }^{18}$ Cycle sequencing was performed on gel purified fragments using the ABI Prism Big Dye Termination kit (Perkin Elmer, Langen, Germany). Sequencing of all 22 mitochondrial tRNA genes, ${ }^{18} A N T 1,{ }^{8}$ Cloorf2 (encoding for Twinkle), ${ }^{11}$ and POLGI ${ }^{15}$ were performed by standard methods. The required primers were designed according to the following Genbank sequences: J04982 for ANT1, AL133215 for C10orf2, and AC005317 for POLG1. In patient 2, the novel F485L mutation was confirmed by restriction fragment length polymorphism (RFLP) analysis of a PCR product containing exon 2 of Twinkle. The mutation F485L introduced a new MseI restriction site. The patient's affected son, her unaffected sister, and 150 control chromosomes were similarly tested for this mutation.

\section{RESULTS}

\section{Morphology and biochemistry of skeletal muscle}

In muscle biopsy specimens stained with Gomori trichrome, both patient 1 and 2 showed ragged red fibres ( 8 and $10 \%$, respectively). These fibres were histochemically negative for
Table 1 Clinical characteristics, structural finding, biochemical and genetic analyses in two patients with adPEO

\begin{tabular}{|c|c|c|c|}
\hline & Patient 1 & Patient 2 & Normal value \\
\hline \multicolumn{4}{|l|}{$\begin{array}{l}\text { Clinical characteristics } \\
\text { and routine analyses }\end{array}$} \\
\hline $\begin{array}{l}\text { Age at onset of PEO/ } \\
\text { gender }\end{array}$ & $15 / M$ & $50 / \mathrm{F}$ & - \\
\hline $\begin{array}{l}\text { Age of onset of systemic } \\
\text { manifestations }\end{array}$ & 49 & 55 & \\
\hline Exercise intolerance & - & + & \\
\hline $\begin{array}{l}\text { Additional clinical } \\
\text { findings }\end{array}$ & $\begin{array}{l}\text { Severe } \\
\text { cardiomyo- } \\
\text { pathy, mild } \\
\text { myopathy }\end{array}$ & $\begin{array}{l}\text { Generalised } \\
\text { myopathy, } \\
\text { axonal } \\
\text { neuropathy, } \\
\text { diabetes, } \\
\text { hearing loss }\end{array}$ & \\
\hline Creatine kinase (U/L) & 32 & 54 & $\begin{array}{l}<80 \text { (men); } \\
<60 \text { (women) }\end{array}$ \\
\hline Electromyography & M & $M / N$ & - \\
\hline \multicolumn{4}{|l|}{ Muscle biopsy } \\
\hline Ragged red fibres (\%) & $8 \%$ & $10 \%$ & - \\
\hline $\begin{array}{l}\text { Mitochondrial } \\
\text { abnormalities on } \\
\text { electron microscopy } \\
\text { Biochemical analysis }\end{array}$ & ND & Yes & \\
\hline $\begin{array}{l}\text { NADH/coQ- } \\
\text { oxidoreductase } \\
\text { (U/g NCP) }\end{array}$ & ND & 22.3 & $12.0-40.0$ \\
\hline $\begin{array}{l}\text { Succinate/cytochrome } \\
\text { c oxidoreductase } \\
\text { (U/g NCP) }\end{array}$ & ND & 9.9 & $6.0-24.0$ \\
\hline $\begin{array}{l}\text { Cytochrome c oxidase } \\
\text { (U/g NCP) }\end{array}$ & ND & 126.0 & $90.0-281.0$ \\
\hline $\begin{array}{l}\text { Citrate synthase } \\
\text { (U/g NCP) }\end{array}$ & ND & 111.0 & $45.0-105.0$ \\
\hline \multicolumn{4}{|l|}{ Genetic analysis } \\
\hline \multicolumn{4}{|l|}{ M+DNA } \\
\hline tRNA genes & Normal & Normal & \\
\hline Southern blot & ND & $\begin{array}{l}\text { Multiple } \\
\text { deletions }\end{array}$ & \\
\hline \multicolumn{4}{|l|}{ Nuclear genes } \\
\hline Sequencing of ANTI & Normal & Normal & \\
\hline $\begin{array}{l}\text { Sequencing of } \\
\text { C1Oorf2 (Twinkle) }\end{array}$ & Normal & F485L & \\
\hline Sequencing of $P O L G 1$ & Normal & Normal & \\
\hline
\end{tabular}

cytochrome c oxidase. Histochemistry of a representative section of muscle from patient $\mathrm{l}$ is shown in fig $2 \mathrm{~A}$.

Biochemical and ultrastructural examinations were performed for patient 2 only. The activities of all RC complexes were within normal ranges per gram of non-collagen protein (NCP) and citrate synthase (CS), but the activity of CS (a mitochondrial marker enzyme) itself was slightly increased (111 U/g NCP; normal range: 45-105) suggesting mitochondrial proliferation. On electron microscopy, ultrastructural abnormalities of mitochondria including paracrystalline inclusions were found (fig 2B).

\section{DNA analysis}

PCR, RFLP, and sequencing analysis of mtDNA tRNA genes in muscle of the two index patients revealed no abnormalities. Southern blot analysis could not be performed in patient 1 because of small sample size. In patient 2, multiple mtDNA deletions were observed on Southern blot. Sequencing of the ANT1, ClOorf2, and POLGl genes in leucocyte DNA did not show any mutations in patient 1 . In patient 2 in the Cloorf2 gene, encoding for Twinkle, a heterozygous missense mutation at nucleotide position C1640A was detected (according to the Genbank accession number AF292004). The mutation exchanged a leucine for a 

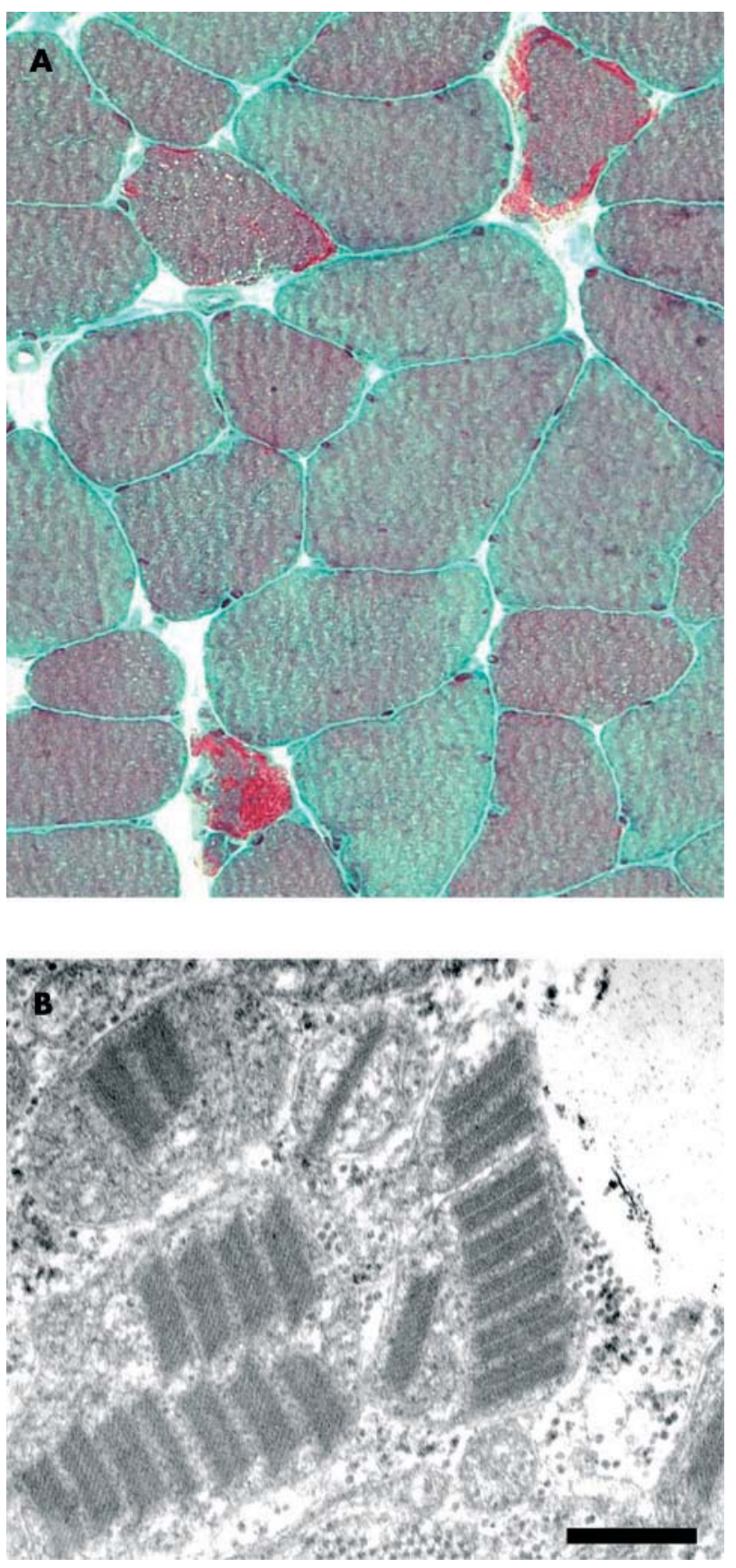

Figure 2 (A) Modified Gomori trichrome staining of a representative section of muscle from patient 1 showing numerous ragged red fibres and mild myopathic changes with increased variability of fibre size and internalised myonuclei, original magnification $\times 20$. (B) Ultrastructure of a muscle fibre from patient 2 containing enlarged mitochondria with crystalloid inclusions (bar $=0.4 \mu \mathrm{m}$ ). The electron micrograph was kindly provided by Professor H Budka, Institute of Neurology, University of Vienna.

conserved phenylalanine at the aminoacid 485 (F485L) (fig 3A). RFLP analysis of Twinkle exon 2 confirmed the mutation in the patient and in her affected son (fig 3B), which was not present in the unaffected sister of the patient or in 150 normal control chromosomes. The mutation is located in a conserved region between Walker motifs A and B of the helicase domain of Twinkle, close to two other previously described pathogenic mutations (W474C and A475P) ${ }^{11}$ and co-segregated with the clinical phenotype in the pedigree (fig 1 ).
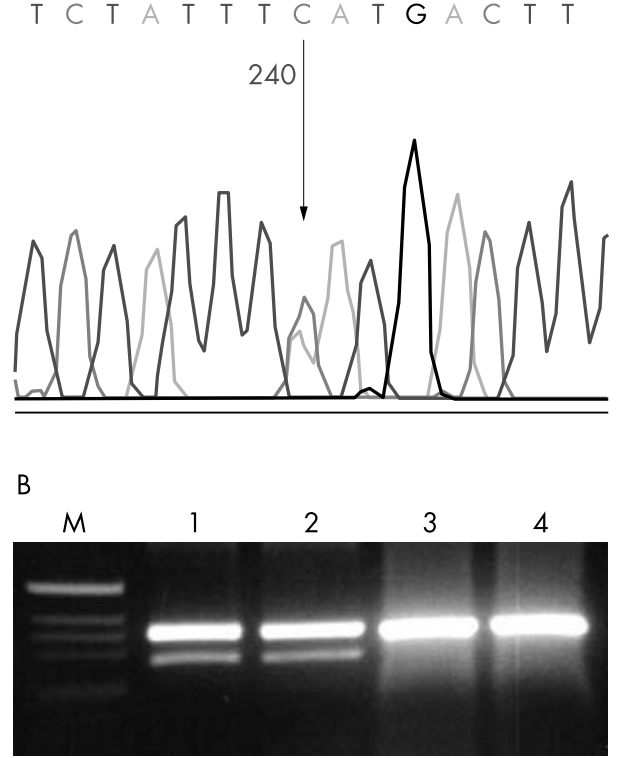

Figure 3 (A) Sequencing of Twinkle revealed a heterozygous missense mutation (arrow). (B) RFLP analysis of Twinkle exon 2 confirmed the mutation in the patient (lane 1) and in her affected son (lane 2), the unaffected sister and a normal control were negative (lane 3, 4). Marker, $1 \mathrm{~kb}$; wild type, $342 \mathrm{bp}$; mutant, $269 \mathrm{bp}$.

\section{DISCUSSION}

We report here two families with dominant transmission of progressive external ophthalmoplegia. In one, a new mutation in Twinkle helicase was identified, whereas the other family had no mutations in the known PEO genes. Presence of multiple mtDNA deletions was verified in patient 2 . Multisystem manifestations and morphological findings in our two families were typical for mitochondrial disease. The first symptom in both patients was PEO, but various other manifestations developed at a later stage of disease (10 to 34 years after onset of PEO) such as severe progressive dilated cardiomyopathy, generalised myopathy, axonal polyneuropathy, diabetes mellitus, and hearing loss. Involvement of extraocular tissues and organs showed high intra-familial variability in both cases. In previous adPEO families, similar systemic manifestations have been described including axonal polyneuropathy, ataxia, depression, dysarthria, tremor, hearing loss, cardiomyopathy, diabetes mellitus, hypogonadism and growth failure. ${ }^{1-16}$ The variety of clinical symptoms closely resembles that seen in mitochondrial encephalomyopathies caused by primary mtDNA mutations, which suggests that acquired mtDNA deletions caused by nuclear gene defects are actually responsible for the tissue dysfunction. Multiple deletions probably arise as somatic mutations and accumulate in non-dividing cells, but do not occur in culturedthat is, dividing, cells from adPEO patients. ${ }^{2}$ This may also explain the late onset and the variable outcome of the disease.

Recent breakthroughs in molecular genetics of PEO allow a more specific genetic classification. Sporadic or maternally inherited forms of PEO are often caused by single mtDNA deletions and several mutations in mitochondrial tRNA genes..$^{20}$ Autosomal disorders of intergenomic communication cause multiple mtDNA deletions or depletion (for review see Moslemi $e t \mathrm{al}^{4}$ ). The estimated frequencies of mutations in adPEO have been reported to be 4-10\% for $A N T 1^{8}$ and $15-35 \%$ for C10orf2 (Twinkle), ${ }^{211}{ }^{15}$ and $45 \%$ for POLG1. ${ }^{14}{ }^{15}$ Mutations in ANT1, Cloorf2 (Twinkle), and POLG1 were recently also described in sporadic patients with PEO. ${ }^{21}$ Cases of adPEO 
still exist in which sequencing or linkage analysis excluded all the three genes as causative. ${ }^{13}$

In both patient 2 and her affected son, a new missense mutation in the gene C10orf 2 encoding Twinkle, a putative mitochondrial ring helicase, was found to change a conserved amino acid at position 485 . This mutation is located between Walker motifs A and B, next to two other previously described pathogenic mutations (W474C and A475P). ${ }^{11}$ Interestingly, in the Twinkle homologue of the T7 bacteriophage, a gene 4 primase helicase, the amino acids corresponding to W474 and A475 in Twinkle are located in a helical loop that contacts the "linker region" of an adjacent subunit in the hexameric complex. Up to date, 8 of 13 published pathogenic mutations cluster in the linker region. ${ }^{11}{ }^{13}$ According to previous functional studies and crystal structure data of the hexameric T7 gene4 protein, this linker region seems to be involved in the multimerisation process and/or to affect catalytic properties of the primase/helicase function. Furthermore, the adjacent subunit interfaces, joined together by the linker helix loop, form the nucleotide binding pocket. Therefore, mutations W474C, A475P, or the newly discovered F485L in the adjacent helical loop could likewise affect subunit interactions or nucleotide binding, or disturb catalytic activities of the complex. Previous functional studies suggest that the multimerisation of the protein is not drastically altered in linker mutants (a 352-364 duplication and W474C), which agrees with the late disease onset, most likely associated with moderate functional defect. ${ }^{10}$

In conclusion, our families illustrate the clinical and genetic variability of adPEO. In one family, sequencing of the gene encoding Twinkle unravelled a novel pathogenic mutation $\mathrm{F} 485 \mathrm{~L}$, whereas in the other index patient with PEO and severe dilated cardiomyopathy, no genetic abnormalities in the ANT1, Cloorf2 (Twinkle), or POLG1 genes could be detected, indicating the existence of a yet unknown nuclear gene defect. Genetic testing has become an important tool in the difficult diagnosis of inherited PEO.

\footnotetext{
Authors' affiliations

S Kiechl, C Thaler, J Wanschitz, J Willeit, Department of Neurology, University Hospital Innsbruck, Innsbruck, Austria

R Horváth, M Jaksch, Metabolic Disease Center Munich-Schwabing and Departments of Clinical Chemistry, Academic Hospital Schwabing, Munich, Germany

P Luoma, A Suomalainen, Department of Neurology and Programme of Neurosciences, Biomedicum Helsinki, University of Helsinki, Finland U Kiechl-Kohlendorfer, Departments of Neonatology, University Hospital Innsbruck, Innsbruck, Austria

B Wallacher-Scholz, Departments of Pediatrics, Academic Hospital Schwabing, Munich, Germany

R Stucka, Gene Centre, Ludwig Maximilian University, Munich, Germany
}

Competing interests: none declared

The first two authors contributed equally to this work

\section{REFERENCES}

1 Zeviani M. Servidei S, Gellera C, et al. An autosomal dominant disorder with multiple deletions of mitochondrial DNA starting at the D-loop region. Nature 1989;339:309-11

2 Suomalainen A, Kaukonen J. Diseases caused by nuclear genes affecting mtDNA stability. Am J Med Genet 2001;106:53-61.

3 Kawai H, Akaike M, Yokoi K, et al. Mitochondrial encephalomayopathy with autosomal dominant inheritance: a clinical and genetic entity of mitochondrial diseases. Muscle Nerve 1995; 18:753-60.

4 Moslemi A-R, Melberg A, Holme E, et al. Autosomal dominant progressive external ophthalmoplegia. Neurology 1999;53:79-84.

5 Carrozzo R, Hirano M, Fromenty B, et al. Multiple mtDNA deletions features in autosomal dominant and recessive diseases suggest distinct pathogeneses. Neurology 1998;50:99-106.

6 Zeviani M, Bresolin N, Gellera C, et al. Nucleus-driven multiple large-scale deletions of the human mitochondrial genome: a new autosomal dominant disease. Am J Hum Genet 1990:47:904-14.

7 Chalmers RM, Brockington M, Howard RS, et al. Mitochondrial encephalopathy with multiple mitochondrial DNA deletions: a report of two families and two sporadic cases with unusual clinical and neuropathological features. J Neurol Sci 1996;143:41-5.

8 Kaukonen J, Juselius JK, Tiranti $\mathrm{V}$, et al. Role of adenine nucleotide translocator 1 in mtDNA maintenance. Science 2000;289:782-5.

9 Napoli L, Bordoni A, Zeviani M, et al. A novel missense adenine nucleotide translocator-1 gene mutation in a Greek adPEO family. Neurology 2001;57:2295-8.

10 Siciliano G, Tessa A, Petrini S, et al. Autosomal dominant external ophthalmoplegia and bipolar affective disorder associated with a mutation in the ANT1 gene. Neuromuscul Disord 2003;13:162-5.

11 Spelbrink JN, Li FY, Tiranti V, et al. Human mitochondrial DNA deletions associated with mutations in the gene encoding Twinkle, a phage T7 gene 4like protein localized in mitochondria. Nat Genet 2001;28:223-31.

12 Moraes CT. A helicase is born. Nat Genet 2001;28:200-1.

13 Lewis S, Hutchison W, Thyagarajan D, et al. Clinical and molecular features of adPEO due to mutations in the Twinkle gene. J Neurol Sci 2002;201:39-44.

14 Van Goethem G, Dermaut B, Lofgren A, et al. Mutation of POLG is associated with progressive external ophthalmoplegia characterized by m+DNA deletions. Nat Genet 2001;28:21 1-12.

15 Lamantea E, Tiranti V, Bordoni A, et al. Mutations of mitochondrial DNA polymerase gamma are a frequent cause of autosomal dominant or recessive progressive external ophthalmoplegia. Ann Neurol 2002;52:21 1-19.

16 Van Goethem G, Martin JJ, Dermaut B, et al. Recessive POLG mutations presenting with sensory and ataxic neuropathy in compound heterozygote patients with progressive external ophthalmoplegia. Neuromuscul Disord 2003; 13:133-42

17 Fischer JC, Ruitenbeek W, Gabreels FJ, et al. A mitochondrial encephalomyopathy: the first case with an established defect at the level of coenzyme Q. Eur J Pediatr 1986;144:441-4.

18 Hofmann S, Bezold R, Jaksch M, et al. Wolfram (DIDMOAD) Syndrome and Leber hereditary optic neuropathy (LHON) are associated with distinct mitochondrial haplotypes. Genomics 1997;39:8-18.

19 Jaksch M, Gerbitz KD, Kilger C. Screening for mitochondrial DNA (mtDNA) point mutations using nonradioactive single strand conformation polymorphism (SSCP) analysis. Clin Biochem 1995;28:503-9.

20 Hirano M, DiMauro S. ANT1, Twinkle, POLG, and TP: New genes open our eyes to ophthalmoplegia. Neurology 2001;57:2163-5.

21 Agostino A, Valletta L, Chinnery PF, et al. Mutations of ANT1, Twinkle, and POLG1 in sporadic progressive external ophthalmoplegia (PEO). Neurology 2003;60:1354-6 